\title{
Time-delay-compensated grating monochromator for FEL beamlines
}

Fabio Frassetto, Elke Ploenjes, Marion Kuhlmann, Luca Poletto

Fabio Frassetto, Elke Ploenjes, Marion Kuhlmann, Luca Poletto, "Timedelay-compensated grating monochromator for FEL beamlines," Proc. SPIE 9210, X-Ray Free-Electron Lasers: Beam Diagnostics, Beamline Instrumentation, and Applications II, 92100 (8 October 2014); doi: 10.1117/12.2065257

SPIE Event: SPIE Optical Engineering + Applications, 2014, San Diego, California, United States 


\title{
Time-delay-compensated grating monochromator for FEL beamlines
}

\author{
Fabio Frassetto ${ }^{\mathrm{a}}$, Elke Ploenjes ${ }^{\mathrm{b}}$, Marion Kuhlmann ${ }^{\mathrm{b}}$, Luca Poletto $^{1 \mathrm{a}}$ \\ ${ }^{a}$ National Council for Research of Italy- Institute of Photonics and Nanotechnologies, via Trasea 7, \\ 35131 Padova, Italy; \\ ${ }^{\mathrm{b}}$ Deutsches Elektronen-Synchrotron DESY, Notkestraße 85, 22603 Hamburg, Germany
}

\begin{abstract}
We present the design of a time-delay-compensated monochromator explicitly designed for extreme-ultraviolet FEL sources, in particular the upcoming FLASH II at DESY (Hamburg). The design originates from the variable-line-spaced (VLS) grating monochromator by adding a second grating to compensate for the pulse-front tilt given by the first grating after the diffraction. The covered spectral range is $6-60 \mathrm{~nm}$, the spectral resolution is in the range 1000-2000, while the residual temporal broadening is lower than $15 \mathrm{fs}$. Accounting for typical FLASH II divergences, the grazing angles on the different optics have been chosen so that the mirrors and gratings are respectively shorter than $500 \mathrm{~mm}$ and $300 \mathrm{~mm}$. The proposed design: 1) minimizes the number of optical elements, since just one grating is added with respect to a standard VLS monochromator B-L; 2) guarantees high focusing properties in the whole spectral range of operation; 3) requires simple mechanical movements, since only rotations are needed to perform the spectral scan.
\end{abstract}

Keywords: Time-delay-compensated monochromator, diffraction, variable-line-spaced grating, free-electron laser

\section{INTRODUCTION}

The consolidation of the FEL sources, from experimental machines to reliable sources, has opened the door to the development of demanding beamlines. In particular, due to the intrinsic multi-harmonics generation process, one of the most demanded BL feature is the possibility to monochromatize the FEL beam even beyond the intrinsic FEL resolution. Inherited from the synchrotron experience, grating monochromators are used in FEL beamlines, both at FLASH [1, 2] and LCLS $[3,4]$. They give both tunability in a broad spectral range and high spectral selectivity.

Unfortunately, especially for operation in the extreme-ultraviolet region of FLASH, the monochromator temporal response strongly affects the FEL pulse duration. As well known, from the expertise gained in the field of the ultrafast optics, the use of a single stage monochromator affects the temporal structure of the selected pulses by introducing a pulse-front tilt due to diffraction $[5,6]$. The temporal broadening can be tolerated in many experiments, nevertheless the full exploitation of the particular temporal characteristics of a FEL source requires the use of a grating monochromator that compensates for the temporal stretch, that we define as Time-Delay-Compensated Monochromator (TDCM). The design consists in using two gratings in a time-delay compensated configuration, where the second grating compensates for the front-tilt and for the spectral spread introduced by the first one [7-13]. TDCMs have been realized and used in high-order laser harmonics beamlines. Pulses as short as 8 to $10 \mathrm{fs}$ have been measured at the output of TDCMs in the 20-45 $\mathrm{nm}$ spectral region [14-17]. The layouts proposed and realized for the high-order laser harmonics beamlines cannot be directly used for FEL pulses, due to the different source parameters, particularly the source peak intensity, and the different requirements, particularly the definitely larger size of the optics.

Here we present the design of a TDCM explicitly tailored for extreme-ultraviolet FEL sources, in particular the upcoming FLASH II, the major extension of FLASH [18]. This substantial upgrade will lead to a new experimental hall and many new beamlines operated in parallel with FLASH. The monochromator design originates from the variable-linespaced (VLS) grating monochromator that is already used at LCLS (see Ref. 3) by adding a second grating to compensate for the pulse-front tilt. The driving parameters for the design are: a) spectral range $6-60 \mathrm{~nm}$; b) spectral resolution in the 1000-2000 range; c) time response shorter than $100 \mathrm{fs}$; d) minimum lateral displacement to let space to

${ }^{1}$ Contact author: poletto@dei.unipd.it, luca.poletto@pd.ifn.cnr.it

X-Ray Free-Electron Lasers: Beam Diagnostics, Beamline Instrumentation, and Applications II, edited by Stefan P. Hau-Riege, Stefan P. Moeller, Makina Yabashi, Proc. of SPIE Vol. 9210 92100I · (c) 2014 SPIE · CCC code: 0277-786X/14/\$18 - doi: 10.1117/12.2065257 
adjacent beamlines; e) minimum vertical displacement to reduce the change of the beam height; f) mirror length shorter than $500 \mathrm{~mm}$; g) grating length shorter than $300 \mathrm{~mm}$.

The main characteristics of the proposed configuration are here resumed as: 1) it minimizes the number of optical elements, since just one grating is added with respect to a standard VLS monochromator beamline; 2) it gives very low displacement of the output beam with respect to the input; 3) it guarantees high focusing properties in the whole spectral range of operation; 4) it requires simple mechanical movements, since only rotations are needed to perform the spectral scan.

\section{ULTRAFAST MONOCHROMATIC BEAMLINE}

\subsection{Beamline design}

To realize a TDCM, two gratings are used in a compensated configuration, i.e., the first grating is demanded to perform the spectral selection on the intermediate slit plane, and the second grating has to compensate for the pulse-front tilt given by the first grating, therefore guaranteeing the ultrafast response. The use of two gratings makes the optical design more complex than a standard monochromator. Aim of the design is to find a simple configuration that is capable to work in compensated design and minimizes the complexity of the optical system.

The concept here presented adopts four optical elements to realize a TDCM for FLASH2. The design originates from the variable-line-spaced (VLS) grating monochromator, that has been proposed by Hettrick [19] and adopted both for synchrotron radiation beamlines [20] and high-order laser harmonics [21, 22]. Recently, the VLS design has been adopted also for the monochromatic beamline at LCLS (see Refs. 3 and 4).

A concave mirror produces a converging beam and a VLS plane grating diffracts the radiation onto the exit slit. The variable groove spacing of the grating provides the additional free parameters to keep the focal distance almost constant as a function of the photon energy and to compensate for high-order aberrations. The VLS monochromator is also rather simple mechanically in that only two optical elements are required and the photon energy is scanned by a single rotation of the grating around an axis passing through its center. The design is adapted to realize a TDCM by adding a second section with an identical VLS plane grating illuminated by the diverging light coming out from the slit and mounted in a compensated geometry. In principle, only three optical elements are required to realize a TDCM, namely the concave mirror and two VLS plane gratings, that are separated by an intermediate slit that carries out the beam monochromatization.

The design has been specialized to the requirements of FLASH II and to its expected performances:

a) Due to the high angular and lateral stability of FLASH II, the monochromator works without an entrance slit, i.e., the FEL itself acts as the source point for the monochromator. In this way, also a pre-focusing mirror is avoided, therefore limiting the number of optical elements. Note that this is also the case of the monochromator at LCLS.

b) Due to the required minimum lateral displacement of the whole beamline with respect to a straight propagation, an additional plane mirror has been inserted between the two gratings to fold the beamline.

c) Due to high photon flux, horizontal and vertical foci have to be kept separated to reduce the radiation density on the slit blades. In particular, the first mirror, that is demanded to illuminate the grating in converging light, is a planeelliptical one. Also the final focusing section is a Kirkpatrick-Baez section consisting of plane-elliptical mirrors.

The optical layout is presented in Fig. 1. The FEL beam is focused by the plane-elliptical mirror M1 toward the plane VLS grating G1. The latter is illuminated in converging light and diffracts the radiation toward the intermediate slit, where the beam is monochromatized. The plane mirror M2 is used to fold the beam, therefore reducing the lateral displacement of the beamline. The grating G2 has the same groove-space-variation parameters as G1 and is mounted symmetrically to $\mathrm{G} 1$ with respect to the slit, to realize the time-delay compensated configuration. The diverging radiation coming out from G2 is finally focused to the sample area by two plane-elliptical mirrors in KB configuration: M4 is focusing the bam in the dispersion plane, M3 in the perpendicular plane.

The use of cylindrical mirrors has been also considered, since they are definitely less expensive than plane-elliptical ones, but the simulations show that, given the actual source divergence, the aberrations introduced by the cylindrical optics compromise the good focusing properties of the beamline.

The whole TDCM beamline consists of four mirrors and two gratings. Only two optical elements, the grating and the plane folding mirror, have been added to obtain the ultrafast response. Note that the folding plane mirror is added to minimize the lateral displacement, since other FEL beamlines are located very close to the monochromatic beamline. In case a larger lateral displacement is tolerated, this additional optical element may be removed. 
With respect to the monochromator beamline at FLASH, which has five mirrors and one grating (see Ref. 1), the total number of optical elements is the same. With respect to the monochromator beamline at LCLS, which has three mirrors and one grating (see Ref. 3), one additional grating and the plane folding mirror have been added.

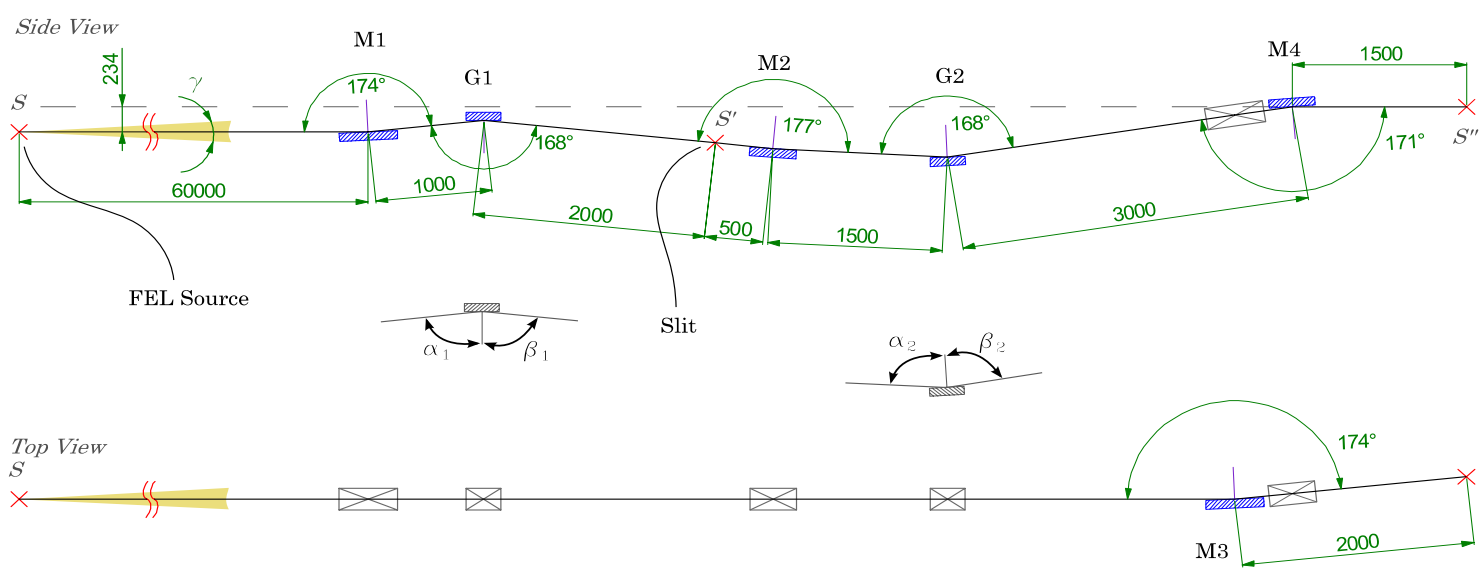

Figure 1. TDCM optical layout. In the side view (the spectral dispersion plane), the exit beam is parallel to the input one.

The incidence angle on the last focusing mirror has been chosen to have the output beam parallel to the input beam in the spectral dispersion plane, that is here assumed as the side view. The final displacement of the output beam with respect to the input is really kept restrained, since the output beam is parallel to the floor, therefore parallel to the entrance in the side view, and at a height that has been increased by about $20 \mathrm{~cm}$. The lateral displacement with respect to the entrance direction in the top view is also about $20 \mathrm{~cm}$.

The direction of the output beam with respect to the horizontal axis may be exchanged just choosing a different incident angle on the final output mirror M4. Given the output direction of the other FLASH II beamlines, the angle on M4 may be selected to have the output of the TDCM beamline parallel to the other beamlines. Therefore, it will be easy to use the same experimental chambers in different beamlines, since all the output axes are expected to be parallel.

The proposed design has the additional advantage that, if the FEL is polarized in the horizontal plane, five of the six optics are illuminated in s-polarization, where the efficiency is higher especially at the long wavelengths.

The source parameters that have been adopted to simulate the beamline performance are listed in Tab. 1.

The optical parameters of the beamline are resumed in Tab. 2. Two sets of gratings are used to cover the $6-60$ $\mathrm{nm}$ spectral region. Given the source divergence as reported in Tab. 1 and the size of the optical elements as reported in Tab. 2, a partial cut of the beam occurs at wavelengths longer than $40 \mathrm{~nm}$. The vignetting is $4 \%$ at

Table 1. Parameters of the FLASH II source. The source divergence has been taken to be $75 \mu \mathrm{rad}$ FWHM @ 40 nm and scaled as $\lambda^{3 / 4}$.

\begin{tabular}{ll}
\hline Source size & $200 \mu \mathrm{m}$ FWHM \\
Source divergence & $18 \mu \mathrm{rad}$ FWHM @ $6 \mathrm{~nm}$ \\
& $27 \mu \mathrm{rad}$ FWHM @ $10 \mathrm{~nm}$ \\
& $45 \mu \mathrm{rad}$ FWHM $@ 20 \mathrm{~nm}$ \\
& $75 \mu \mathrm{rad}$ FWHM @ $40 \mathrm{~nm}$ \\
& $102 \mu \mathrm{rad}$ FWHM $@ 60 \mathrm{~nm}$ \\
\hline
\end{tabular}
$40 \mathrm{~nm}, 6 \%$ at $50 \mathrm{~nm}, 20 \%$ at $60 \mathrm{~nm}$.

\subsection{Optical performance}

The optical performances have been simulated by a ray-tracing program that includes the capability of calculating the pulse-front tilt after the grating diffraction. The wavelength scanning is performed by the rotation of the two gratings around an axis passing through its center. G1 is operated in the internal spectrum, i.e., $\beta \mathrm{G} 1<\alpha \mathrm{G} 1$, to have the incident angle on G1 higher than $84^{\circ}: 85.0^{\circ}<\alpha \mathrm{G} 1<87.3^{\circ}$. On the contrary, G2 is operated in the external spectrum to realize a compensated configuration, i.e., $\beta \mathrm{G} 2>\alpha \mathrm{G} 2$, which results in $80.7^{\circ}<\alpha \mathrm{G} 2<83.0^{\circ}$. The use of G1 at incident angles higher than $85^{\circ}$ makes it safer to operate the grating under the intense FEL beam. G2 is illuminated at incident angles 
lower than $83^{\circ}$, but being it located after the intermediate slit, which carries out the monochromatization, the FEL beam intensity on $\mathrm{G} 2$ is much lower than at G1.

Table 2. Parameters of the TDCM beamline

\begin{tabular}{|c|c|c|}
\hline \multicolumn{3}{|c|}{ Distances } \\
\hline $\mathrm{S}-\mathrm{M} 1$ & $\mathrm{~m}$ & 60 \\
\hline $\mathrm{M} 1-\mathrm{G} 1$ & $\mathrm{~m}$ & 1 \\
\hline M1 - Slit (S') & $\mathrm{m}$ & 2 \\
\hline $\mathrm{M} 2-\mathrm{G} 2$ & $\mathrm{~m}$ & 1.5 \\
\hline $\mathrm{G} 2-\mathrm{M} 3$ & $\mathrm{~m}$ & 2.5 \\
\hline M3 - M4 & $\mathrm{m}$ & 0.5 \\
\hline M4 - S', & $\mathrm{m}$ & 1.5 \\
\hline \multicolumn{3}{|c|}{ Mirrors } \\
\hline \multicolumn{3}{|c|}{ M1 } \\
\hline Type & & Plane / Elliptical \\
\hline Entrance Arm & $\mathrm{m}$ & 60 \\
\hline Exit Arm & $\mathrm{m}$ & 3 \\
\hline Incidence Angle & deg & 87 \\
\hline Coated Area & $\mathrm{mm} \times \mathrm{mm}$ & $500 \times 40$ \\
\hline \multicolumn{3}{|c|}{$\mathrm{M} 2$} \\
\hline Type & & Plane \\
\hline Incidence Angle & deg & 88.5 \\
\hline Coated Area & $\mathrm{mm} \times \mathrm{mm}$ & $400 \times 40$ \\
\hline \multicolumn{3}{|c|}{$\mathrm{M} 3$} \\
\hline Type & & Plane / Elliptical \\
\hline Entrance Arm & $\mathrm{m}$ & 67.5 \\
\hline Exit Arm & $\mathrm{mm}$ & 2000 \\
\hline Incidence Angle & deg & 87 \\
\hline Coated Area & $\mathrm{mm} \times \mathrm{mm}$ & $500 \times 30$ \\
\hline \multicolumn{3}{|c|}{ M4 } \\
\hline Type & & Plane / Elliptical \\
\hline Entrance Arm & $\mathrm{mm}$ & 5000 \\
\hline Exit Arm & $\mathrm{mm}$ & 1500 \\
\hline Incidence Angle & deg & 85.5 \\
\hline Coated Area & $\mathrm{mm} \times \mathrm{mm}$ & $400 \times 25$ \\
\hline
\end{tabular}

\begin{tabular}{|c|c|c|}
\hline \multicolumn{3}{|c|}{ Gratings } \\
\hline \multicolumn{3}{|c|}{ G1-A G2-A } \\
\hline Type & & VLS Plane \\
\hline Spectral Region & $\mathrm{nm}$ & $6-20$ \\
\hline $\begin{array}{l}\text { Central groove } \\
\text { density }\end{array}$ & $\mathrm{mm}^{-1}$ & 600 \\
\hline Deviation Angle & deg. & 168 \\
\hline Ruled coated area & $\mathrm{mm} \times \mathrm{mm}$ & $250 \times 20$ \\
\hline \multicolumn{3}{|c|}{ G1-B G2-B } \\
\hline Type & & VLS Plane \\
\hline Spectral Region & $\mathrm{nm}$ & $18-60$ \\
\hline $\begin{array}{l}\text { Central groove } \\
\text { density }\end{array}$ & $\mathrm{mm}^{-1}$ & 200 \\
\hline Deviation angle & deg & 168 \\
\hline Ruled coated area & $\mathrm{mm} \times \mathrm{mm}$ & $300 \times 40$ \\
\hline
\end{tabular}

The FWHM resolution on the intermediate slit is shown in Fig. 2 for a 50 -um-wide slit. It has been calculated as $\lambda / \Delta \lambda$, where $\lambda$ is the wavelength and $\Delta \lambda$ the FWHM bandwidth that is transmitted through the slit. The resolution is higher than 1000 in the whole spectral range of operation, according to the requirements.
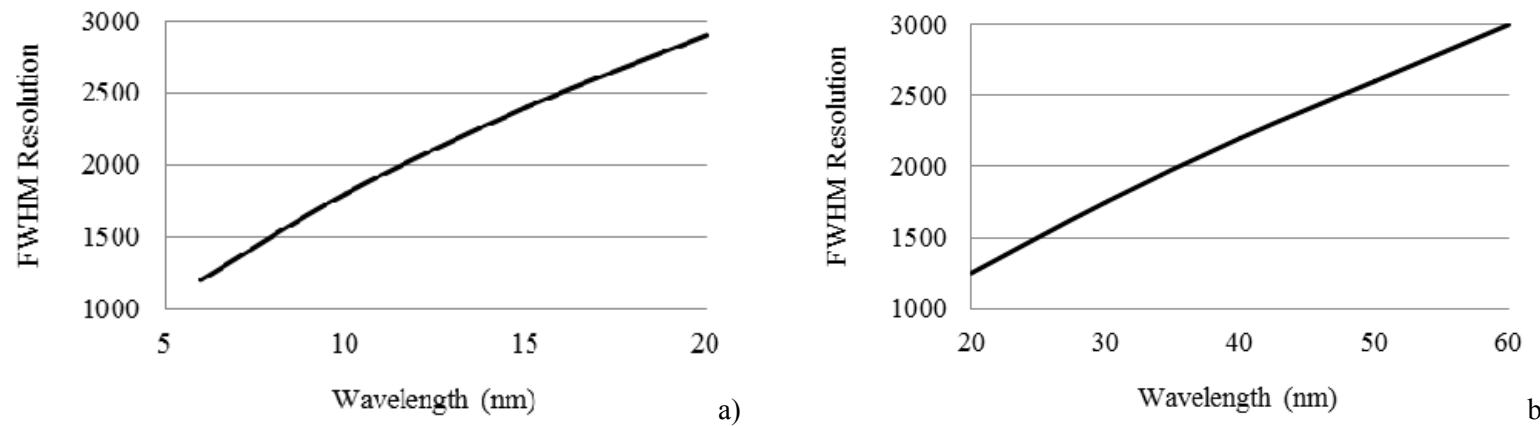

Figure 2. FWHM resolution, $\lambda / \Delta \lambda$ : a) $600 \mathrm{gr} / \mathrm{mm}$ grating; b) $200 \mathrm{gr} / \mathrm{mm}$ grating. 
The FWHM pulse-front tilt is shown in Fig. 3 in the intermediate plane and in Fig. 4 at the output of the monochromator. The compensated configuration is really effective, being able to compensate for the front-tilt from the picosecond time scale down to few femtoseconds. The temporal resolution of the beamline is increased by almost three orders of magnitudes.

Finally, the FWHM spot size is shown in Fig. 5, being in the 10-15 um range, and so compatible with the typical experimental requirements at a generic FEL target area.
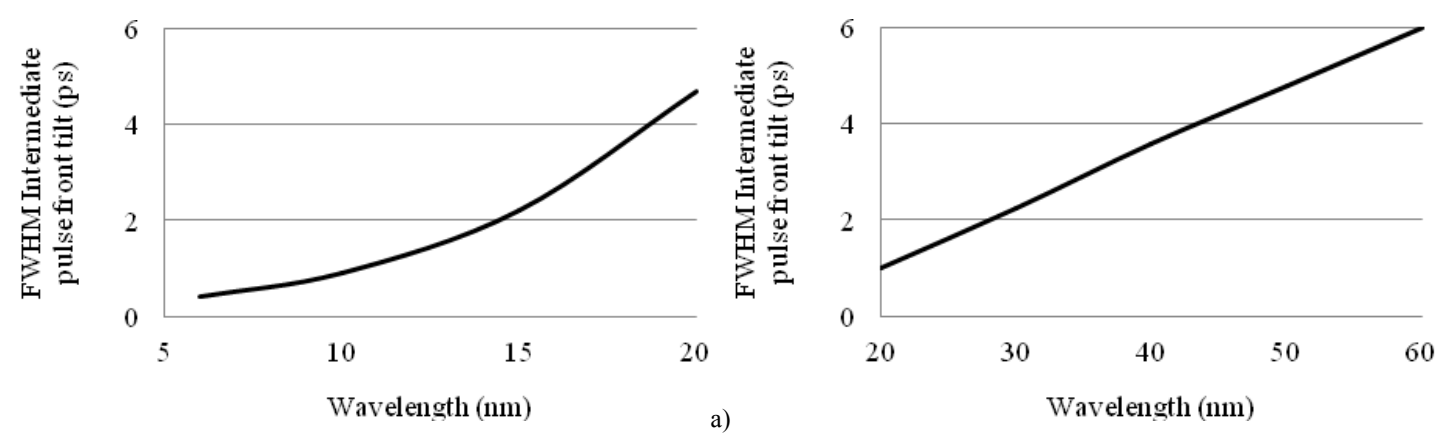

Figure 3. FWHM pulse-front tilt at the intermediate plane: a) $600 \mathrm{gr} / \mathrm{mm}$ grating; b) $200 \mathrm{gr} / \mathrm{mm}$ grating.
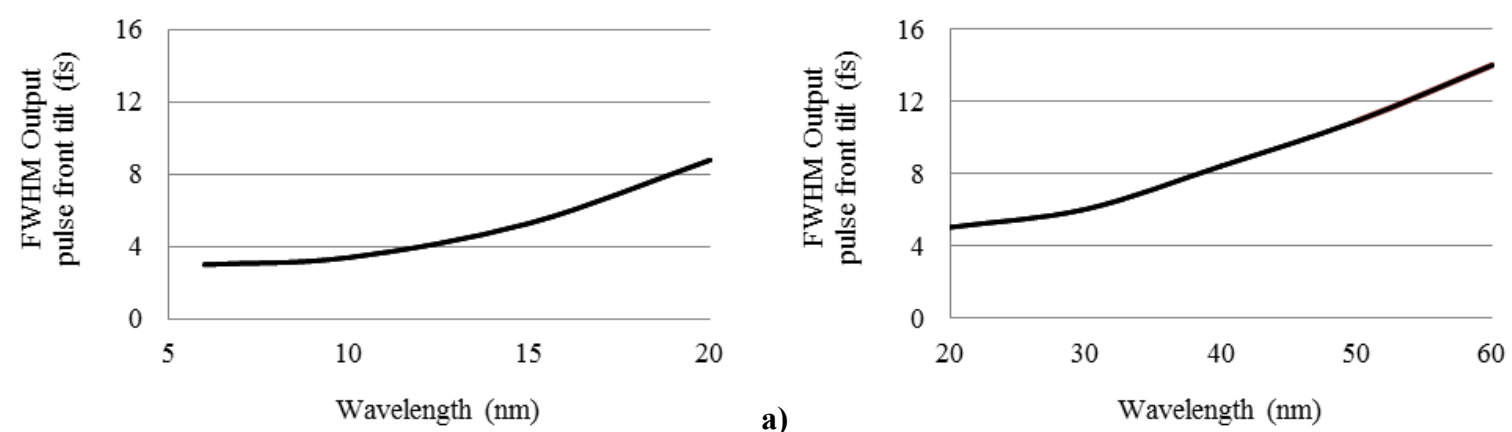

Figure 4. FWHM pulse-front tilt at the output: a) $600 \mathrm{gr} / \mathrm{mm}$ grating; b) $200 \mathrm{gr} / \mathrm{mm}$ grating.
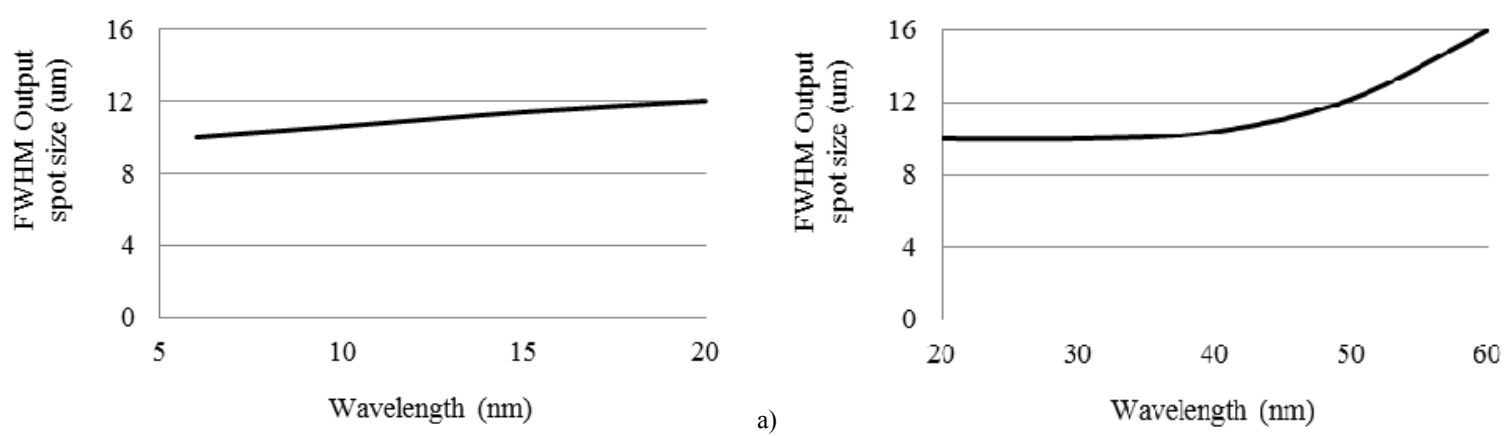

Figure 5. FWHM spot size in the focal plane: a) $600 \mathrm{gr} / \mathrm{mm}$ grating; b) $200 \mathrm{gr} / \mathrm{mm}$ grating. 


\subsection{Efficiency}

The total efficiency of the beamline is mainly determined by the efficiency of the gratings, since there are two of them. The efficiency curve are strongly dependent on the available grating profiles. At present, VLS gratings for the extremeultraviolet are realized with laminar profile. As an example, the efficiency curve of the VLS plane grating (provided by Jobin-Yvon, France) that has been used to realize the spectrometer-monochromator described in Ref. 20 for the spectral region 5-30 nm is shown in Fig. 6. Taking the values shown in Fig. 6, the efficiency of the TDCM beamline is expected to be 5-15 times lower than the efficiency of a standard VLS grating beamline.

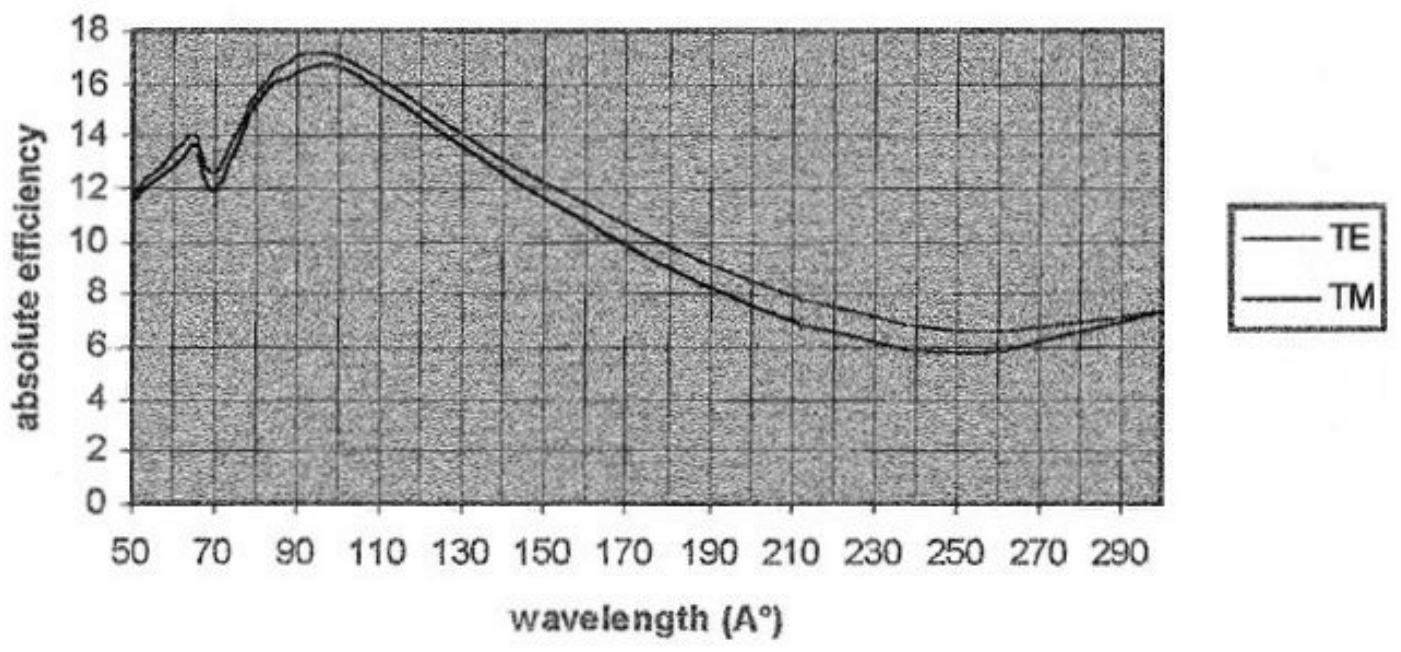

Figure 6. Efficiency curve of a plane VLS grating (provided by Jobin-Yvon) used to realize a spectrometer-monochromator for high-order laser harmonics. The central groove density is $600 \mathrm{gr} / \mathrm{mm}$, the profile is laminar with groove height of $20 \mathrm{~nm}$ and duty cycle of $60 \%$. The coating is gold.

\section{CONCLUSIONS}

We have presented the design of a TDCM explicitly tailored for extreme-ultraviolet FEL sources, in particular the upcoming FLASH II. The configuration here presented shows that a TDCM beamline may be realized in a relatively simple configuration, extending the VLS-plane-grating monochromator configuration to the use with ultrashort pulses in the extreme-ultraviolet region. Only six optical elements are required: four mirrors and two gratings. Despite the necessity of operate two gratings in the compensated configuration, the mechanical complexity is very low: only two rotations are required to perform the spectral scan. Furthermore, the temporal resolution of the beamline is increased by almost three orders of magnitudes with respect to a single-grating beamline.

The proposed configuration minimizes the number of optical elements, since just one grating is added with respect to a standard VLS monochromator beamline, gives very low displacement of the output beam with respect to the input, and guarantees high focusing properties in the whole spectral range of operation.

It is worth to note that, although being tailored to the FLASH II requirements, the proposed concept can be extended to any extreme-ultraviolet FEL.

\section{REFERENCES}

[1]M. Martins, M. Wellhöfer, J. T. Hoeft, W. Wurth, J. Feldhaus and R. Follath, "Monochromator beamline for FLASH," Rev. Sci. Inst. 77, 115108 (2006)

[2] N. Guerasimova, S. Dziarzhytski, J. Feldhaus, "The monochromator beamline at FLASH: performance, capabilities and upgrade plans," J. Mod. Opt. 58, 1480 (2011)

[3]P. Heimann, O. Krupin, W. F. Schlotter, J. Turner, J. Krzywinski, F. Sorgenfrei, M. Messerschmidt, D. Bernstein, J. Chalupský, V. Hájková, S. Hau-Riege, M. Holmes, L. Juha, N. Kelez, J. Lüning, D. Nordlund, M. F. Perea, A. Scherz, R. 
Soufli, W. Wurth and M. Rowen, "Linac Coherent Light Source soft x-ray materials science instrument optical design and monochromator commissioning," Rev. Sci. Inst. 82, 093104 (2011)

[4]W. F. Schlotter, J.J. Turner, M. Rowen, P. Heimann, M. Holmes, O. Krupin, M. Messerschmidt, S. Moeller, J. Krzywinski, R. Soufli, M. Fernández-Perea, N. Kelez, S. Lee, R. Coffee, G. Hays, M. Beye, N. Gerken, F. Sorgenfrei, S. Hau-Riege, L. Juha, J. Chalupsky, V. Hajkova, A.P. Mancuso, A. Singer, O. Yefanov, I.A. Vartanyants, G. Cadenazzi, B. Abbey, K.A. Nugent, H. Sinn, J. Lüning, S. Schaffert, S. Eisebitt, W.-S. Lee, A. Scherz, A. R. Nilsson, and W. Wurth, "The soft x-ray instrument for materials studies at the linac coherent light source x-ray free-electron laser," Rev. Sci. Inst. 83, 043107 (2012)

[5]L. Poletto and F. Frassetto, "Time-preserving grating monochromators for ultrafast extreme-ultraviolet pulses," Appl. Opt. 49, 5465 (2010)

[6]F. Frassetto and L. Poletto, "Grating monochromators for the spectral selection of femtosecond extreme-ultraviolet pulses," Nucl. Inst. Meth. A 635, S75 (2011)

[7]P. Villoresi, "Compensation of optical path lengths in extreme-ultraviolet and soft-x-ray monochromators for ultrafast pulses," Appl. Opt. 38, 6040 (1999)

[8]L. Nugent-Glandorf, M. Scheer, D.A. Samuels, V. Bierbaum, S.R. Leone, "A laser-based instrument for the study of ultrafast chemical dynamics by soft x-ray-probe photoelectron spectroscopy," Rev. Sci. Inst. 73, 1875 (2002)

[9]J. Norin, K. Osvay, F. Albert, D. Descamps, J. Yang, A. L'Huillier, C. Wahlstrom, "Design of an extreme-ultraviolet monochromator free from temporal stretching," Appl. Opt. 43, 1072 (2004)

[10] L. Poletto, "Time-compensated grazing-incidence monochromator for extreme-ultraviolet and soft X-ray high-order harmonics," Appl. Phys. B 78, 1013 (2004)

[11] L. Poletto and P. Villoresi, "Time-compensated monochromator in the off-plane mount for extreme-ultraviolet ultrashort pulses," Appl. Opt. 45, 8577 (2006)

[12] L. Poletto, "Tolerances of time-delay compensated monochromators for extreme-ultraviolet ultrashort pulses," Appl. Opt. 48, 4526 (2009)

[13] L. Poletto, F. Frassetto and P. Villoresi, “Ultrafast Grating Instruments in the Extreme Ultraviolet,” J. Sel. Top. Quant. Electron. 18, 467 (2012)

[14] L. Poletto, P. Villoresi, E. Benedetti, F. Ferrari, S. Stagira, G. Sansone, and M. Nisoli, "Intense femtosecond extreme ultraviolet pulses by using a time-delay compensated monochromator," Opt. Lett. 32, 2897 (2007)

[15] L. Poletto, P. Villoresi, F. Frassetto, F. Calegari, F. Ferrari, M. Lucchini, G. Sansone, M. Nisoli, "Time-delay compensated monochromator for the spectral selection of extreme-ultraviolet high-order laser harmonics," Rev. Sci. Instrum. 80, 123109 (2009)

[16] M. Ito, Y. Kataoka, T. Okamoto, M. Yamashita, and T. Sekikawa, "Spatiotemporal characterization of single-order high harmonic pulses from time-compensated toroidal-grating monochromator," Opt. Express 18, 6071 (2010)

[17] H. Igarashi, A. Makida, M. Ito, T. Sekikawa, "Pulse compression of phase-matched high harmonic pulses from a time-delay compensated monochromator," Opt. Express 20, 3725 (2012)

[18] E. Plönjes, B. Faatz, J. Feldhaus, M. Kuhlmann, K. I. Tiedtke, R. Treusch, "FLASH2 beamline and photon diagnostics concepts,” Proceedings of FEL2013 - New York, WEPSO50, 614 (2013)

[19] M. Hettrick and S. Bowyer, "Variable line-space gratings: new designs for use in grazing incidence spectrometers," Appl. Opt. 22, 3921 (1983)

[20] J. H. Underwood and J. A. Koch, "High-resolution tunable spectrograph for x-ray laser linewidth measurements with a plane varied-line-spacing grating," Appl. Opt. 36, 4913 (1997)

[21] L. Poletto, G. Tondello, and P. Villoresi, "Optical design of a spectrometer-monochromator for the extremeultraviolet and soft-x-ray emission of high-order harmonics," Appl. Opt. 42, 6367 (2003)

[22] L. Poletto, S. Bonora, M. Pascolini and P. Villoresi, "Instrumentation for analysis and utilization of extremeultraviolet and soft X-ray high-order harmonics,” Rev. Sci. Instr. 75, 4413 (2004) 